\title{
Strain Rate and Temperature Effects on the Non Linear Behaviour and the Damage Process Induced by Tensile Loading within RTPMMA «Rubber Toughened Polymethylmethacrylate»
}

\author{
Y. Ibouchichene-Aiche and J.-L. Lataillade \\ Laboratoire Matériaux Endommagement Fiabilité (ENSAM), Esplanade des Arts et Métiers, \\ 33405 Talence-Bordeaux, France
}

\begin{abstract}
RÉSUMÉ:
Les polymères choc, comme matériaux composites obtenus par incorporation de particules, sont dédiés à des application structurelles grâce à leur comportement rendu ainsi ductile. Cet article décrit une méthode ayant pour but de caractériser l'endommagement dans ce type de matériau aux grandes vitesses d'allongement atteignant $10^{3} \mathrm{~s}-1$. Cette technique consiste à soumettre une éprouvette à double partie utile à un essai de traction dynamique moyennant des dispositifs à chargement par impact. L'évolution qualitative de l'endommagement est effectuée par une approche macroscopique. les résultats présentés ici sont obtenus aux faibles vitesses, et sont confrontés à ceux obtenus avec une autre technique utilisée par l'équipe SCHIRRER [1]. La comparaison montre que la méthode, présentée ici, est très prometteuse pour la caractérisation de l'endommagement. Par ailleurs, l'influence de la température sur le comportement non linéaire du matériau est montrée.
\end{abstract}

\begin{abstract}
:
Rubber toughened polymers "RTP", when they are used in structural elements to ensure absorption energy gives better results than those of glassy polymers which generally behave in brittle manner. The aim of this work is to investigate the strain rate and temperature effects on the non lineaire behaviour of RTPMMA. Furthermore, we used a macroscopical approach to characterize the damage process induced by tensile loading on a special dumbell shape specimen. Compared to the results obtained by an other technique used by SCHIRRER [1], this method seems to be a promising one to the characterization of damage.
\end{abstract}

\section{1-INTRODUCTION :}

Several experimental studies showed that the incorporation of rubber paricles, by mechanical blending, in a glassy polymer matrix improve the fracture toughness and the absorption of a large amount of energy resulting from plastic deformation during impact. In 1995 O.JULIEN showed the influence of the volume fraction of the modifying particles and their morphology on the fracture toughness (pure rubber particles, core-shell rubber particles,...) $[10$ - 12]. It has been demonstrated that the most efficient toughning effect is for volume fraction of about $40 \%$ [2-5] and for the the particles nature influence, high toughness was observed in three-layer particle systems compared with two layer ones [6].

In other respect, recent studies demonstrate that the mechanical damage mechanisms may be triggered by the cavitation of the rubber content in the particles $[1,7]$. More recently it was showed by SCHIRRER [1] using volume change, light absorption and light scattering that three types of microstructural damage exists in RTPMMA ; pure matrix plasticity at very low strain rates $\left(10^{-5} \mathrm{~s}^{-1}\right)$, rubber cavitation at medium strain rates and disordered cavitation rubber tearing and matrix plasticity at high strain rates $\left(10^{-1} \mathrm{~s}-1\right)$. Using the theory of coherent light backscattering SCHIRRER [8] found that the damage process starts by the cavitation of the rubber particles. This cavitated particle changes rapidly from its original state to a stable destroyed state with $8 \%$ void content; then this phenomena grows by successive particle destructions, whereas the void content of individual damaged particle remains constant. As the aim of this present work is to investigate the influence of strain rates and temperature on the mechanical properties of the same material used by SCHIRRER $[1,8]$, it appeared important to us to investigate a wide range of testing rates $\left[10^{-4}\right.$. $\left.10^{4} \mathrm{~S}^{-1}\right]$. This mechanical properties like yield stress seems to be considerably strain rate and temperature dependent [9]. We try therefore to interprate the non linear tensile yield behavior of RTPMMA using the REE-EYRING theory. To characterize the damage process, here a new procedure is applied which is based on the use of a special dumbell shape specimens. Since this technique is dedicated to the damage tests at very high strain rates $\left(10^{4} \mathrm{~S}^{-1}\right.$ ), it appears important to us to test it at very low strain rates and to compare the results to those obtained with an other damage testing method used by SCHIRRER [1] 


\section{2- EXPERIMENTAL TECHNIQUES:}

\section{2-1 Material :}

The matrix of the polymer studied here is a low molecular weight PMMA "Poly Methyl Methacrylate" ( $\overline{\mathrm{M}_{\mathrm{W}}}=120000$ ), which has been toughned with $40 \%$ of core-shell rubber particles. The crosslinked rubber shell component is based on Butyl Acrylate Styrene, and is about $15 \%$. The average external particle diameter is $200 \mathrm{~nm}$ (see figure 1). This material was synthesised by ATOHAAS company and referred to Altuglas EI-CH 50. The normelised tensile modulus is approximately $2000 \mathrm{MPa}$.

To impove the bright parts state, all the specimens used for this work have been machined using a laser beam $((\lambda=10.6 \mu)$.

\section{2-2 Failure tests:}

To investigate the effects of strain rate and temperature on the RTPMMA behavior the non linear tensile yielding process has been investigated over several decades of strain rates at temperatures in the range $\left(-20^{\circ}\right.$ $-90^{\circ} \mathrm{C}$ ). The yield point is taken as the well defined maximum exhibited in all the tensile curves.(see figure

At low strain rates we used a static tensile machine "INSTRON"; whereas at medium strain rates we have used a high velocity servo-hydraulic testing machine ZWICK REL. This apparatus consists to give a constant velocity to a hydraulic jack which tests specimens under tensile loading. The sample, as shown in figure 3, was a simple dumbell specimen; the entire length width, length and thickness are respectively about 10,30 and $2 \mathrm{~mm}$.

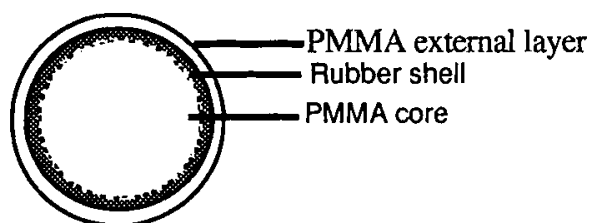

Figure 1: Core-shell nubber particle

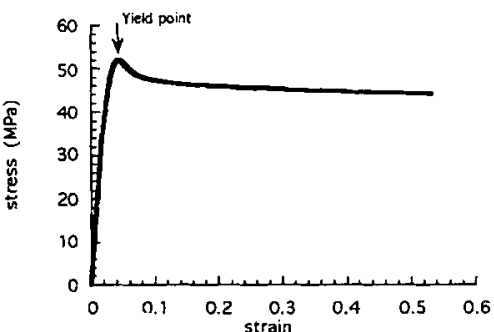

Figure 2: RTPMMA behaviour

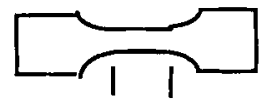

entire length $=30 \mathrm{~mm}$

Figure 3: Simple dumbell specimen

\section{2-3 Damage tests:}

With the aim of investigating the effects of high strain rates on the damage process induced within RTPMMA by tensile loading, a new method is proposed here and evaluated at low strain rate. It is based on the use of special specimens containing two entire length (see figure 4 ). One of them is slightly weakented (its cross section is smaller then the other one) and plays a role of a fuse . 
The failure of this fuse allows us to load the investigated specimen itself at a given strain below its fracture strain; in this way we can control its damage degree.

The change of the fuse cross section let us to damage the specimens at different strain levels. These specimens have been tested with a static tensile machine "ADAMEL LHOMARGY DY 36". To evaluate this damage we use a macroscopical approach which consists in a qualitative mesure using an internal conventionel parameter " $E$ : elastic longitudinal modulus". This parameter has been chosen to evaluate the changes in the inner structure of RTPMMA during deformation process.

After the fuse breakage the entire length damaged yet is loaded again under static conditions in order to evaluate the elastic modulus $E_{D}$ and therefore the damage according to the following formula $\mathrm{D}=\left(\mathrm{E}_{0}-\mathrm{ED}_{\mathrm{D}}\right) / \mathrm{E}_{0}$ where $\mathrm{E}_{0}$ is the elastic modulus of undamaged specimen.

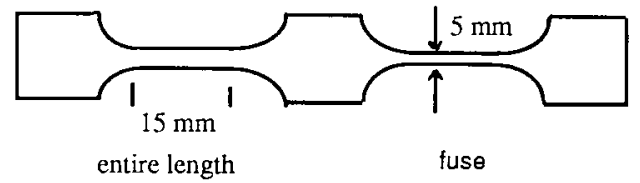

Fig 4: The simple dedicated to damage tests

The results deduced from this technique were compared to those we have obtained using an other technique developped by SCHIRRER [1], which consists on the use of a special software generating a constant cross head speed with 30 equaly spaced small unloadings at constant high speed. The damage parameter D is defined by the same formula mentionned previously and the elastic modulus $\mathrm{E}$ is derived from slight unloadings.(see figure 5)

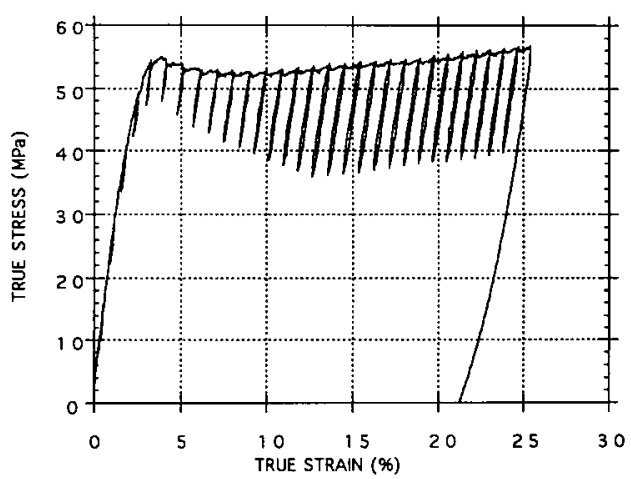

Fig 5 : True stress versus true strain with small unloadings at $\dot{\varepsilon}=0.04 \mathrm{~s}-1$

\section{3- EXPERIMENTAL RESULTS and DISCUSSION:}

\section{3-1 Mechanical properties:}

Experimental results confirm a strong strain rate and temperature dependence of the main parameters characterizing this material. In fact a plot of the yield stress against the logarithm of strain rate at several constant temperatures point out a critical strain rate $\left(\dot{\varepsilon}_{c}\right)$ depending on the temperature (Figure 6). 
Unfortunately it was not possible to extend the strain rate for covering values up $10^{2} \mathrm{~S}^{-1}$ with this technique-But the results obtained show the existence of two regimes; the transition between them is related to the competition of two deformation modes required to free different kinds of molecular motions implied in the deformation process. The plastic flow at the yield point is attributed to the matrix plasticity and therefore the thermal activation energy is associated to its secondary relaxations.

We tryed to interprate this yield behaviour using the REE-EYRING theory illustrated by the following formula:

$$
\frac{\left|\sigma_{\text {ecoul }}\right|}{T}=A_{\alpha}\left(\operatorname{Ln} 2 C_{\alpha} \dot{\varepsilon}+\frac{Q_{\alpha}}{R T}\right)+A_{\beta} \sinh ^{-1}\left(C_{\beta} \dot{\varepsilon} \frac{Q_{\beta}}{R T}\right)
$$
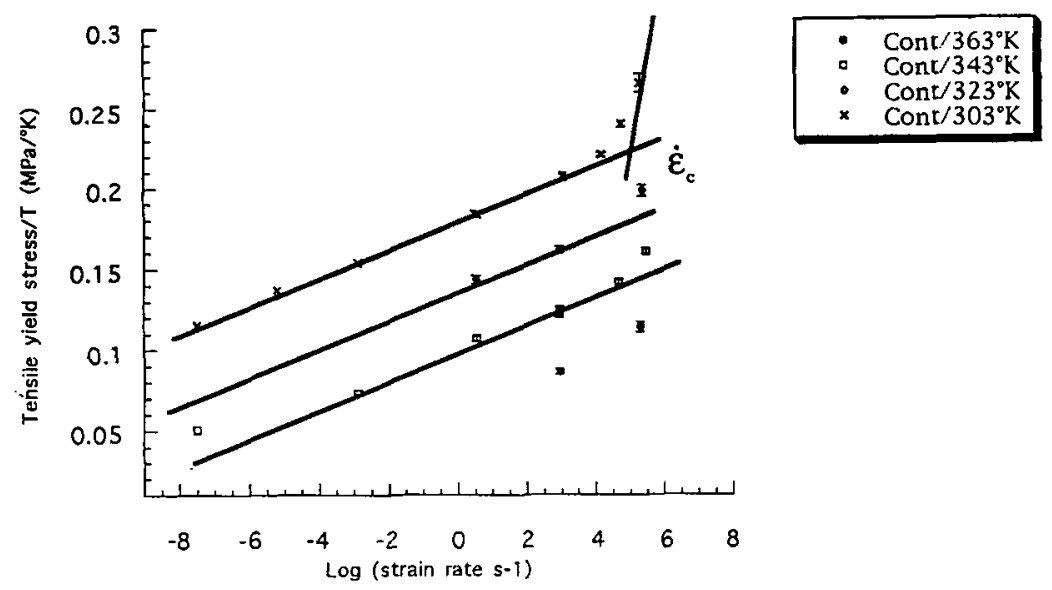

Figure 6: Ratio of tensile yield to temperature as a function of the logarithm of strain rate

If one assumes that two rate processes are involved in the deformation at yield, we can evaluate in this work (through the above theory) the activation energy to $109 \mathrm{Kcal} / \mathrm{mol}$. Since this value is close to that one of pure PMMA calculated by BAUWENS-CROWET [9], it is therefore related to the relaxation of the matix. Using the following formula $(\mathrm{Va}=\mathrm{kT} / \partial \sigma / \partial \ln \dot{\varepsilon})$ the activation volume was also calculated and the results are summarized in table 1

\begin{tabular}{|c|c|c|c|c|}
\hline $\mathrm{T}\left({ }^{\circ} \mathrm{K}\right)$ & 303 & 323 & 343 & 363 \\
\hline $\mathrm{Va}\left(\mathrm{A}^{3}\right)$ & 1320 & 1406 & 1493 & 1580 \\
\hline
\end{tabular}

Table 1: Activation volume Va at different temperatures

At low temperatures, around $-20^{\circ} \mathrm{C}$, the brittle fracture of the RTPMMA occurs by multifragmentation and absence of whitening. The last phenomena reveals that the damage process is negligible. The figure 7 shows the brittleness of material when the strain rate is rising, whereas in figure 8 we have plotted the evolution of the yield point against the temperature. A tansition brittle/ductile is observed around $300^{\circ} \mathrm{K}$. 


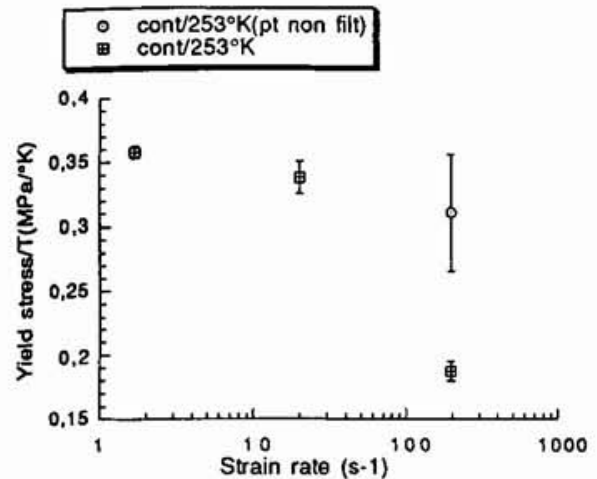

Fig 7:yield point evolution versus strain rate at $\mathrm{T}=-20^{\circ} \mathrm{C}$

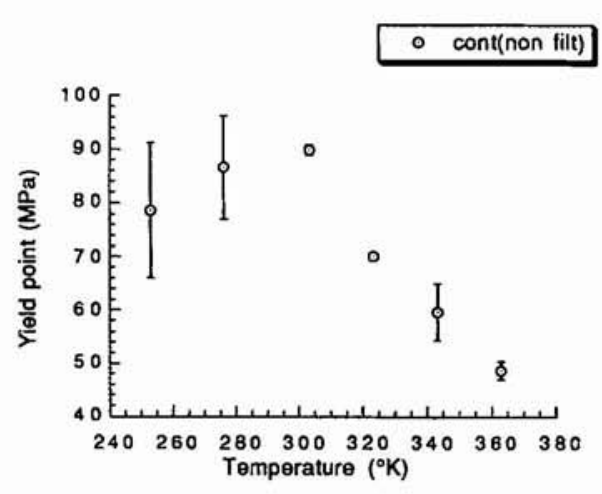

Fig 8: Yield point evolution versus temperature at $\dot{\varepsilon}=200$ S-1

\section{3-2 Damage results:}

Before reaching the yield point, the damage process starts by appearence of whitening and at the end of the test the specimen becames opaque. From the results obtained by this technique SCHIRRER we have plotted in the figure 9 the evolution of the damage parameter $D$ in respects with true strain ; and we have compared in the figure 10 the results obtained by the two methods at $\dot{\varepsilon}=0.11 \mathrm{~S}-1$ and $0.04 \mathrm{~S}-1$.

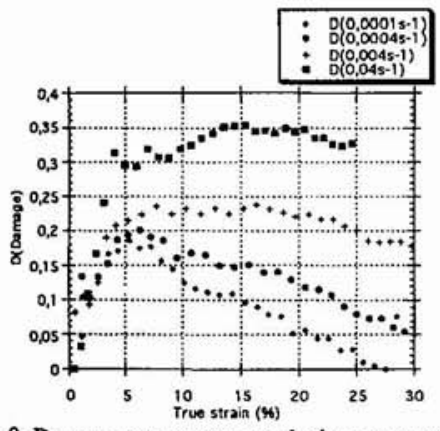

Fig 9: Damage parameter evolution versus true strain rate

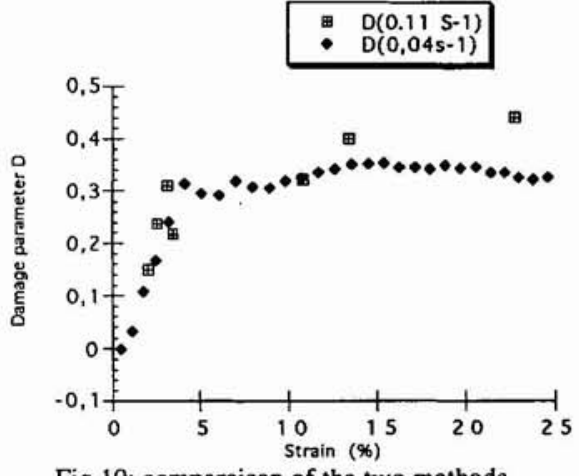

Fig 10: comparaison of the two methods

The figure 11 represents the double entire length specimen after test

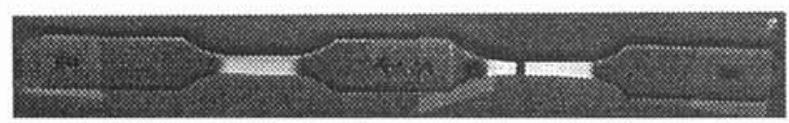

The initiation and propagation of the damage process seems also affected by the strain rate. The damage increase from a threshold value until a maximum one which depends on the strain rate, it decreases after or even reaches zero at higher strains At these strains, tensile moduli increase due to molecular orientation in the load direction thus the material becomes strongly anisotropic. Therefore, the decrease in modulus due to damage can be compensated by anisotropy. 
Damage in RTPMMA appears to be a voiding process which starts at low strains of the order of $4 \%$ and which by diffraction of light causes the whitening of the structure.

\section{4- CONCLUSION :}

In conclusion, it appears that the behaviour at yield of RTPMMA is very sensitive to the strain rate and temperature effects. In fact it is possible to explain the modifications in this behaviour using the REEEYRING model by knowing the matrix and reiforcement's principals relaxations; So the interpretation given yet in the above paragraph require a validation by DMA mesurements which is qualified to point out these relaxations.

Furthermore, the damage study by means of interrupted loading tests appears to be reliable for investigating the progressive damage mechanisms within RTPMMA. The damage technique described above, allows us to define a relation coupling the elastic properties of RTPMMA with the damage level. It is hence easy to us to investigate the influence of strain rate and temperature on the damage process induced by impact loading.

\section{REFERENCES:}

[1] : R.SCHIRRER, C.FOND, A. LOBBRECHT. "Volume change and light scattering during mechanical damage in PMMA toughened with core shell rubber particles" . To be published in JMS.

[2] : O. JULIEN , PH. BEGUELIN, L. MONNERIE , H.H. KAUSCH.

"The laoding rate dependence of the fracture behavior of rubber modified poly methyl methacrylate ", will be published in "Rubber toughening II 1995 "

[3] : O.MAUZAC, R.SCHIRRER, J.Mat. Sci. 25 , (1987) 5125-5133

[4] : J.M.GLOAGUEN, P.STEER, P.GAILLARD, C.WROTECKI, J.M.Lefevre, Pol. Eng and Sci.33 (1993), 12, 748-753.

[5] : C.B.BUCKNALL, I.K. PARTRIDGE, M.V.WARD, J. Mat.Sci. 19, (1984) 2064-2072.

[6] : C.J.HOOLEY, D.R.MOORE,M.WHALE, M.J.WILLIAMS, Plastics and Rubber Processing and Applications, 1, 4, (1981), 345-349

[7] : C.FOND, A.LOBBRECHT, R.SCHIRRER, Polymers toughened with rubber microspheres : an analytical solution for stresses and strain in the rubber at equilibrium and rupture. (1996) to be published in Internalional Journal of Fracture.

[8] : R.SCHIRRER, R. LENKE, J. BOUDOUAZ. Study of Mechanical Damage in Rubber Toughened PMMA by Single and Multiple Scattering of Light. To be published in P.E.S .

[9] :C. BAUWENS-CROWET "The compression yield behaviour of polymethylmethacrylate over a wide range of temperature and strain rates". J. Mater Sci 8(1973) 968-979

[10] : C.J.G.PLUMMER, PH.BEGUELIN, H.-H.KAUSCH " On the influence of particle morphology on microdeformation in rubber modified POLY(METHYL METHACRYLATE)". Polymer Vol.37 No.1, pp.7$10,1996$.

[11] : PH.BEGUELIN, "Approche experimentale du comportement mécanique des polymères en sollicitation rapide", Thèse No 1572 LAUSANNE, EPFL (1996).

[12] : O. MAUZAC, "Approche locale de la fissuration des polymères choc amorphes", Thèse STRASBOURG (1990). 